\title{
FATORES de PROTEÇÃo E DE RISCO ASSOCIADOS AO INÍCIO DO USO DE CANNABIS: REVISÃO SISTEMÁTICA
}

\author{
Tânia Moraes Ramos Andrade ${ }^{1}$; \\ Sérgio de Paula Ramos ${ }^{2}$
}

\begin{abstract}
Este artigo teve por objetivo verificar os fatores associados ao início do uso de cannabis, através de revisão sistemática, utilizando as bases MEDLINE, SciELO, LILACS e Cochrane, com os descritores: cannabis, marijuana, onset, de 1999 a abril de 2008. Os resultados obtidos para os fatores associados à iniciação de cannabis foram: uso precoce do álcool e tabaco, gênero masculino, comportamento agressivo e desviante, baixo monitoramento parental, pais solteiros, uso de substâncias pelos pais, grupo de pares com uso de cannabis, pertencente a comunidades desfavorecidas. O uso precoce da cannabis é fator preditor para transtorno de uso de substâncias psicoativas em jovens adultos.
\end{abstract}

Descritores: Cannabis; Marijuana; Comportamento.

\footnotetext{
${ }_{1}^{1}$ Psicóloga, Mestre em Psicologia, Professor Assistente, Faculdade de Psicologia, Pontifícia Universidade Católica do Rio Grande do Sul, RS, Brasil. Professor Assistente, Faculdade de Psicologia, Universidade Luterana do Brasil, Canoas, RS, Brasil. E-mail: taniaandrade@terra.com.br.

2 Psiquiatra, Doutor em Psiquiatria, Unidade de Dependência Química, Hospital Mãe de Deus, Porto Alegre, RS, Brasil.
} 


\section{Protection AND RISK FACTORS ASSOCIATED TO INITIAL USE OF CANNABIS: SYSTEMATIC REVIEW}

This article aims to identify factors associated with the onset of cannabis use through a systematic review using Medline, Scielo, Lilacs and Cochrane, with the following keywords: cannabis, marijuana, onset, from 1999 to April 2008. The results for factors associated with the initiation of cannabis were: early alcohol and tobacco use; male gender; aggressive and deviant behavior; low parental monitoring; single parents; use of substances by parents; group of peers using cannabis; belonging to disadvantaged communities. The early use of cannabis is a predictive factor for Substances Use Disorders (SUD) in young adults.

Descriptors: Cannabis; Marijuana; Behavior.

\section{LOS FACTORES DE PROTECCIÓN Y RIESGO ASOCIADOS AL USO INICIAL DEL CANNABIS: REVISIÓN SISTEMÁTICA}

La finalidad de ese artículo es examinar los factores asociados al uso inicial de cannnabis, a través de una revisión sistemática: Medline, Scielo, Lilacs y Cochrane, con las siguientes palabras clave: cannabis, marijuana, inicio, desde el 1999 hasta abril del 2008. Los factores siguientes se asocian al inicio del cannabis: uso temprano del alcohol y del tabaco; género masculino; comportamiento agresivo temprano; supervisión parental baja; padres solos; uso de sustancias de los padres; grupo de pares que usan cannabis; comportamiento irregular; el pertenecer a las comunidades perjudicadas. El uso temprano del cannabis es un factor predictivo para los desordenes del uso de las sustancias (SUD) por los adultos jóvenes.

Descriptores: Cannabis; Marijuana; Comportamiento.

\section{Introdução}

Adolescência

A Organização das Nações Unidas (ONU) define como jovens as pessoas entre 15 e 24 anos de idade, enquanto isso, a Organização Mundial de Saúde (OMS) refere-se à adolescência como processo fundamentalmente biológico que vai dos 10 aos 19 anos de idade, abrangendo a pré-adolescência (de 10 a 14 anos) e a adolescência propriamente dita (de 15 a 19 anos). Já a juventude é considerada categoria sociológica que implica na preparação dos indivíduos para o exercício da vida adulta, compreendendo a faixa dos 15 aos 24 anos de idade. No Brasil, para o Estatuto da Criança e do Adolescente (ECA), a infância acontece até os 12 anos de idade e a adolescência dos 12 aos 18 anos.

$\mathrm{Na}$ adolescência, se tudo transcorrer bem, o jovem atingirá a vida na plenitude de todos os seus potenciais, dispondo de substrato orgânico, afetivo, emocional e cognitivo para se desenvolver ${ }^{(1)}$. Porém, se nesse processo falharem fatores intrínsecos (biológicos, genéticos e emocionais) e/ou extrínsecos (família, escola, amigos e a comunidade), a transformação poderá ser interrompida em diferentes níveis e graus de complexidade.

Os adolescentes representam a população mais estudada em relação ao uso de drogas, sendo que, nas últimas décadas, foram realizados inúmeros levantamentos epidemiológicos sobre o uso de drogas entre os adolescentes. Esses levantamentos contribuem para a avaliação de realidades específicas e regionalizadas tanto no contexto internacional como no nacional ${ }^{(2)}$. Recentemente, em 
2010, foi realizado o I Levantamento Nacional sobre Uso do Álcool, Tabaco e Outras Drogas entre universitários das 27 capitais brasileiras ${ }^{(3)}$. Foram entrevistados 18.000 estudantes com idade entre 18 e 25 anos. Esse estudo revelou que quase metade dos universitários brasileiros, isto é, $48,7 \%$ consumiram pelo menos uma vez na vida alguma substância psicoativa, exceto álcool e tabaco, e $26,1 \%$ referiram ter utilizado cannabis pelo menos uma vez na vida. Comparando os dados norte-americanos com os brasileiros, para o uso de cannabis, o consumo de cannabis é maior entre os universitários norte-americanos.

\section{Uso de substâncias psicoativas na adolescência}

Os dados do National Institute on Drug Abuse ${ }^{(4)}$ mostram que 25 milhões de americanos, com 12 anos ou mais, tinham feito uso de cannabis pelo menos uma vez no último ano. A partir desses dados, surgiram inúmeros levantamentos com adolescentes americanos, como o estudo $^{(5)}$ que informa o uso de substâncias psicoativas em cada série do High School. Os dados revelam aumento do uso de cannabis nas séries finais da escola secundária, isto é, dos adolescentes que usaram cannabis no último ano, $10,3 \%$ cursavam o oitavo ano, $24,6 \%$ cursavam o décimo ano e $31,7 \%$ estavam no décimo segundo ano. Dos adolescentes que utilizaram cannabis pelo menos uma vez na vida, $11,2 \%$ estavam no oitavo ano, $15,9 \%$ no décimo ano e $24,9 \%$ no décimo segundo ano. Em relação ao uso de substâncias psicoativas, sabe-se que a incidência do consumo tem aumentado na adolescência ${ }^{(6-7)}$, e, em vista disso, pesquisadores brasileiros também têm se ocupado desse tema ${ }^{(8-12)}$.

De acordo com o levantamento nacional sobre consumo de drogas psicotrópicas ${ }^{(8)}, 22,6 \%$ do total estimado de estudantes brasileiros da rede estadual de ensino fizeram uso de drogas. Quanto ao uso na vida de cannabis, o índice é de 5,9\%. Porto Alegre, comparando-se com outras capitais brasileiras, apresentou a maior prevalência de uso na vida de cannabis, $10,9 \%$. Outro importante dado observado foi que os estudantes que haviam feito uso na vida de substâncias psicoativas apresentaram maior defasagem escolar, quando comparados aos estudantes que não fizeram uso dessas substâncias.

No que diz respeito ao índice de maior uso de cannabis na cidade de Porto Alegre, comparado a outras capitais brasileiras, estudos regionais corroboram e contribuem para o levantamento desses resultados, como se pode apontar no estudo ${ }^{(11)}$ que investigou 1.586 estudantes do ensino fundamental e médio de escolas públicas e particulares, onde se pode verificar o índice para uso na vida de cannabis de $21,1 \%$. A prevalência de uso de cannabis e inalantes foi equivalente entre os gêneros.

Também, em outro estudo ${ }^{(12)}$ com 706 estudantes de nível médio, os resultados obtidos para o uso na vida de cannabis, entre os adolescentes, foi de $20,7 \%$. Os pesquisadores ressaltam que a idade média de iniciação do uso do álcool e de tabaco pelos adolescentes é menor do que a idade do início do uso de cannabis e outras drogas. Esses achados, em relação à iniciação, também são referidos em outros estudos ${ }^{(8,11,13)}$, verificando-se aumento linear na idade da experimentação, de acordo com cada substância.

Fatores associados ao início do uso de cannabis

É importante salientar que os fatores associados, abordados nesta revisão, dizem respeito tanto aos fatores de risco como aos fatores protetores para o início do uso de cannabis. Assim como refere o estudo ${ }^{(13)}$ que avaliou 6.417 estudantes de escolas públicas de São Paulo, incluindo estudantes de $5^{\text {a }}$ série do ensino fundamental ao $3^{\circ}$ ano do ensino médio. Os resultados sugerem que os fatores que estavam associados para o início do uso de drogas são: baixo rendimento escolar, pobre relacionamento com as pessoas com as quais reside, estudar no período da tarde, presença de comportamento antissocial, problemas familiares e possuir amigos que usam drogas.

Vale destacar os resultados obtidos de importante pesquisa $^{(14)}$ que monitorou inicialmente crianças com quatro meses de idade até completarem 21 anos. Os índices investigados referem que o uso precoce de cannabis é fator preditor para, mais tarde, ocorrer dependência de cannabis.

No que diz respeito à variável idade ${ }^{(7)}$, essa foi associada fortemente ao risco de desenvolver transtorno de uso de substância psicoativa, isto é, quanto menor a idade de início de uso para o álcool e cannabis, maior a probabilidade de risco para desenvolver a dependência. Aqueles com idade de 14, 16, 17 e 18 anos tiveram risco aumentado quando comparadas a outros com idade entre 22 e 26 anos, para desenvolver dependência de cannabis.

Examinando a associação entre a frequência do uso de cannabis e as consequências psicossociais na adolescência e no jovem adulto, estudo relevou ${ }^{(6)}$ que o uso regular e o uso pesado de cannabis aos 14 e 15 anos, comparados ao uso recente, entre 20 e 21 anos, estiveram associados a aumento da taxa de problemas de desajustamento como: o uso de outras drogas ilícitas, crime, depressão e comportamento suicida.

Aocorrência de determinadas circunstâncias na vida de uma criança ou adolescente pode aumentar a probabilidade de que venha desenvolver comportamento de uso de álcool, tabaco ou outras drogas ${ }^{(15)}$. Essas circunstâncias são fatores de risco que, através de inúmeros estudos, têm demonstrado associação com o uso de substâncias. Por outro lado, os fatores de proteção podem diminuir a probabilidade de que essa criança ou adolescente venha a se envolver com o uso de drogas. Cabe salientar que tanto o fator de risco quanto o de proteção não determina o aparecimento de comportamento ou patologia e, sim, aumenta ou diminui a probabilidade do evento ocorrer. Os fatores de risco são classificados como presentes ou ausentes nos seguintes grupos: no próprio indivíduo, na família, na escola, entre os pares e na comunidade.

Os fatores de risco e proteção podem afetar os adolescentes em diferentes fases da adolescência ${ }^{(16)}$. Devese observar que quanto mais o adolescente se expuser aos fatores de risco, mais esse adolescente poderá usar drogas de abuso. Alguns riscos na adolescência atuam mais fortemente que os outros, e uma maneira de prevenção 
do início do uso é equilibrar o fator de risco com fator protetor, por exemplo, a pressão que o grupo de pares exerce no adolescente deve ser equilibrada com adequado vínculo entre pais e filhos. A mudança ocorre através de intervenções na família, escola e comunidade.

Considerando a alta prevalência do uso de substâncias psicoativas entre os adolescentes, torna-se necessário investigar alguns fatores associados ao uso de drogas, particularmente a cannabis, no intuito de proporcionar mais informações para serem utilizadas na implementação de programas de prevenção tanto primária e secundária como terciária.

\section{Objetivo}

Este estudo teve como objetivo realizar revisão sistemática sobre os fatores associados ao início do uso de cannabis. Cabe ressaltar que os fatores associados são tanto os fatores de risco como os fatores protetores quanto ao início do uso de cannabis.

\section{Método}

O método realizado foi de revisão sistemática, utilizando, para busca, as bases computadorizadas
MEDLINE, SciELO, LILACS e Cochrane, com os seguintes descritores: cannabis, marijuana, onset, no período de 1999 a abril de 2008.

Foram incluídos artigos empíricos e de revisão com amostras de adolescentes, não importando o delineamento dos mesmos. Os estudos selecionados deveriam avaliar os fatores de iniciação ao uso de cannabis. Foram excluídos os artigos de prevalência, tratamento, comorbidades e a progressão do uso de cannabis.

Nessa busca, foram identificadas 321 referências, cujos resumos foram checados em busca de artigos potencialmente elegíveis, resultando em 35 artigos completos que foram avaliados de acordo com os critérios estabelecidos e, desses, foram selecionados 14 artigos que investigaram os fatores associados ao início do uso de cannabis.

\section{Resultados}

Os 14 artigos obtidos, por meio de revisão sistemática, apresentaram dados coletados em relação ao tipo de estudo e delineamento, país de procedência, número de participantes, idade média dos participantes, dados esses resumidos na Tabela 1 .

Tabela 1 - Descrição de artigos incluídos na revisão

\begin{tabular}{|c|c|c|c|c|c|}
\hline Autor & Tipo de estudo & Monitoramento & País & Número da amostra & Idade média \\
\hline Brook et al. ${ }^{(16)}$ & LO & 13 anos & EUA & 976 & $3-26$ \\
\hline Coffey et al. ${ }^{(17)}$ & LO & 3 anos & Austrália & 2.032 & $14-15$ \\
\hline Wills et al. ${ }^{(18)}$ & TV & & EUA & 1.810 & 11 \\
\hline Von Sydow et al. ${ }^{(19)}$ & LO & 4 anos & Alemanha & 2.446 & $14-24$ \\
\hline \multirow[t]{3}{*}{ Chen et al. ${ }^{(20)}$} & TV & & Panamá & 12.589 & 16 \\
\hline & & & América Central & & \\
\hline & & & República Dominicana & & \\
\hline Patton et al. ${ }^{(21)}$ & LO & & EUA/Austrália & 5.769 & $10-15$ \\
\hline Chen et al. ${ }^{(22)}$ & LO & 2 anos & EUA & 2.311 & 15 \\
\hline Best et al. ${ }^{(23)}$ & TV & & Inglaterra & 2.078 & $14-16$ \\
\hline Hayatbakhsh et al. ${ }^{(24)}$ & LO & 21 anos & Austrália & 3.008 & $0-21$ \\
\hline Agrawal et al. ${ }^{(25)}$ & $\mathrm{CO}$ & 5 anos & EUA & 3.729 & 21 \\
\hline Guxens et al. ${ }^{(26)}$ & LO & 4 anos & Espanha & 1.056 & 12 \\
\hline Guxens et al. (27) & Revisão sistemática & & Espanha & & \\
\hline Agrawal et al. ${ }^{(28)}$ & $\mathrm{CO}$ & 10 anos & EUA & 1.065 & $13-19$ \\
\hline Iglesias ${ }^{(29)}$ & TV & & Chile & 54.000 & $8-21$ \\
\hline
\end{tabular}

TV - Estudo transversal

LO - Estudo longitudinal

Em 1999, foi realizado o primeiro estudo ${ }^{(17)}$ que verificou os fatores preditores do início do uso de cannabis, nas fases da adolescência (pré-adolescência, adolescência, adolescência tardia e jovem adulto). A amostra foi de 976 adolescentes, testados com idade de 13, 15, 21 e 26 anos. Pode-se perceber que a idade de início do uso de cannabis teve aumento quase linear entre 12 e 17 anos, com pico aos 17 anos e, a partir dessa idade, ocorreu diminuição de iniciação de uso até os 25 anos de idade. Cabe salientar que o início do uso de cannabis ocorreu em cada idade estudada (pré-adolescência, adolescência, adolescência tardia e a partir dos 20 anos). Os meninos utilizaram mais cannabis do que as meninas. Os autores sugerem que esse resultado esteja associado aos acessos mais favoráveis ao consumo de cannabis pelos meninos. As variáveis preditoras quanto ao início do uso de cannabis foram: personalidade, grupo de pares e família. Constatou-se também, no estudo, que as maiores taxas de iniciação foram de adolescentes que tinham comportamento despreocupado e livre, possuíam grupo de pares que usavam tabaco e cannabis ou eram 
apenas favoráveis ao uso dessas duas substâncias, e de adolescentes que tinham pais usuários de tabaco e parentes que usavam drogas. Em contrapartida, pais calorosos e filhos identificados com os valores dos pais, tiveram baixa taxa de iniciação em cannabis.

Por meio de dados obtidos em outro estudo(18), que examinou os fatores preditores para o início do uso de cannabis e a progressão do uso, identificou-se como fator de risco para o início de uso de cannabis, entre os adolescentes de 44 escolas secundárias: o uso do tabaco, o uso do álcool, adolescentes que tinham condutas antissociais, adolescentes que possuíam pares que usavam drogas e adolescentes, filhos de pais separados. Em relação à progressão do uso, o aumento nas taxas de uso, na série central secundária, foi fator preditor para o aumento do uso na série final secundária. A transição do uso ocasional na série central foi preditor para o uso regular de cannabis na série final. Esse índice foi encontrado em 12\% dos usuários, mais nos meninos do que nas meninas, sendo o grupo de pares fator determinante para a progressão do uso.

Outra variável estudada nas pesquisas foi a relação entre o temperamento e o uso de substâncias ${ }^{(19)}$, isto é, a dimensão do temperamento (bom controle de self e pior controle de self), associado ao início precoce de substâncias (tabaco, álcool e cannabis). Os resultados mostram que os adolescentes que tinham bom controle de self demonstraram maior competência acadêmica, menor uso de substâncias e, igualmente, grupo de pares com menor uso de substâncias. Entre os adolescentes com pior controle de self estavam adolescentes com mais eventos negativos de vida e mais pares desviantes. Os autores concluem que esses achados oferecem muitas implicações para os programas de prevenção, pois a intervenção precoce é fator protetor contra o início de uso.

Em outro estudo ${ }^{(20)}$, com 2.446 adolescentes entre 14 e 24 anos, examinados aos 14-15 anos, 16-21 e 22-24 anos, foi verificado que os fatores de risco da incidência do início do uso, do abuso e da dependência de cannabis foram: a pressão para o uso de substâncias pelo grupo de pares, a disponibilidade da droga, ter atitudes favoráveis ao uso futuro da droga, baixa autoestima, história familiar (transtornos mentais, morte dos pais antes dos 15 anos, relacionamento prejudicado com um dos pais) e experiências anteriores com drogas lícitas.

No que diz respeito à prática religiosa e ao envolvimento com drogas (álcool, tabaco, cannabis), pode-se citar o estudo(21) em que participaram 12.589 estudantes de 1.656 escolas. Os dados indicaram que ter prática religiosa é inversamente associado ao uso precoce de tabaco e da experiência com cannabis. Jovens sem religião possuíam 2-3 mais chances de uso de cannabis, comparados aos jovens católicos. As conclusões desse estudo referem que prática religiosa é fator de proteção ao uso precoce de tabaco e cannabis, mas esse comportamento não influencia o jovem na decisão de consumir a droga, uma vez que já tenha tido oportunidade de uso.

Apenas um estudo ${ }^{(22)}$, nesta revisão, especificou a etapa da puberdade, investigando os estágios da puberdade e a associação quanto ao início do uso e abuso de cannabis. Foram entrevistados 5.769 estudantes, com idade entre 10 e 15 anos que cursavam a $5^{\mathrm{a}}, 7^{\mathrm{a}}$ e $9^{\mathrm{a}}$ série. Os achados demonstraram que o estágio central da puberdade esteve associado ao aumento duas vezes maior de uso na vida de substâncias psicoativas, e o estágio de puberdade tardia esteve associado com três vezes mais aumento de uso na vida comparado ao estágio central da puberdade e com o estágio inicial. O estágio central esteve associado a modesto aumento no uso recente, e o estágio de puberdade tardia esteve associado com duas vezes mais aumento. Em relação aos abusadores, a iniciação foi três vezes maior na puberdade tardia do que na fase da puberdade central. Assim, pode-se concluir que a fase da puberdade tardia apresentou maior ocorrência de cannabis tanto de uso na vida como uso recente e entre os abusadores.

Outro ponto relevante, evidenciado nas pesquisas, foi o monitoramento parental durante a escola primária e sua influência quanto ao risco do uso de cannabis $^{(23)}$. Os resultados desse estudo indicaram que as crianças que tiveram menos monitoramento de seus pais na escola primária, comparadas com aquelas que tinham melhor monitoramento, possuíam mais chances de experimentar cannabis. Bem como o comportamento coersivo adotado pelos pais esteve associado, moderadamente, ao aumento do risco de exposição para o uso de cannabis pelos filhos. Esses dados sugerem que as práticas parentais de monitoramento, nas primeiras séries, podem exercer forte e duradoura influência na diminuição do risco para experimentação de cannabis.

No que diz respeito aos fatores sociais, familiares e protetores, relacionados ao uso de cannabis, foi verificado em uma pesquisa ${ }^{(24)}$ com 2.078 adolescentes entre 14 e 16 anos, de escolas secundárias, que $24 \%$ dos adolescentes tinham feito uso na vida de cannabis e $15 \%$ tinham usado cannabis no último mês. Os fatores preditores de início de uso foram: possuir amigos e vizinhança que usavam álcool e tabaco, adolescentes que se envolviam em atividades criminais e adolescentes que gastavam parte do seu tempo livre mais com amigos do que com os seus pais. Entre os que tinham usado cannabis, dois fatores foram preditores para iniciação: o uso precoce e a frequência de uso. Os resultados obtidos são que o início precoce, a frequência de uso e a rede social desfavorável são fatores preditores para a iniciação do uso.

É importante ressaltar que um entre quatro australianos, na idade de 14 anos, havia experimentado cannabis pelo menos uma vez na vida. Paralelamente a essa incidência de uso de cannabis, pesquisadores verificaram também que ocorriam mudanças na estrutura familiar australiana (divórcios, aumento do número de segundo e subsequente casamento), além disso, aumento de famílias que adotavam crianças. A partir desses dados, surgiram estudos que examinaram a associação entre as mudanças do status marital e o início do uso de cannabis pelos adolescentes ${ }^{(25)}$. Nesse estudo, foram selecionadas 3.008 mães e seus filhos, desde o nascimento até completarem 21 anos de idade. As entrevistas eram realizadas no terceiro e quinto dia após o parto e quando seus filhos completavam seis meses, 5, 
14 e 21 anos. Os resultados obtidos mostraram que existe associação do estado marital materno e o início precoce do uso de cannabis, isto é, as mudanças do status marital da mãe, quando a criança está entre 5 e 14 anos, estiveram associadas significativamente ao aumento do risco de uso de cannabis. Os pesquisadores ressaltam que não é o status marital que influencia os adolescentes a usarem cannabis, mas a extensão do impacto da mudança marital que a criança vivencia.

O objetivo desse estudo foi ${ }^{(26)}$ investigar, especificamente nas meninas, o uso do tabaco, álcool e cannabis. Os resultados obtidos, em ordem de ascensão da escalada do uso, foi primeiramente o uso do tabaco, o álcool, o uso de cannabis e, posteriormente, outra droga. Pode-se observar que o uso na vida de cada substância esteve associado ao uso de substâncias subsequentes. Por exemplo, o uso precoce do álcool (menor de 16 anos) esteve associado ao uso precoce do tabaco. O uso de cannabis esteve associado ao tabaco (12 anos) e álcool (14 anos), sendo que o uso precoce e a escolha de uso de múltiplas substâncias estiveram associados à classe da substância subsequente.

O consumo de cannabis na Espanha foi examinado primeiramente em estudo com 1.056 estudantes, de 44 escolas secundárias ${ }^{(27)}$. Os fatores identificados para a iniciação incluem consumo de tabaco e álcool, comportamento antissocial, baixo rendimento escolar, padrão de tempo livre, uso de drogas pelos pares, tipo de escola e situação familiar. Como os fatores preditores diferem largamente em idade e gênero, os pesquisadores analisaram o consumo de substância psicoativa em cada ano escolar. A verificação desses índices foi realizada em quatro segmentos, no primeiro segmento foram coletados índices da $7^{\mathrm{a}}$ série, no segundo segmento dados da $8^{\text {a }}$ série, no terceiro segmento dados da $9^{\text {a }}$ série e, no quarto segmento, dados da $10^{\text {a }}$ série. Esses resultados também foram separados por gênero. Foi verificado, em relação aos meninos, que as variáveis como: cursar a $7^{\mathrm{a}}$ série, consumir tabaco, possuir comportamento de risco para o consumo de álcool (ficar bêbado, tomar quatro ou mais doses no mesmo evento), ter grupo de pares que consumiam cannabis e comportamento antissocial (matar aula, brigar, quebrar coisas, pegar objetos de outras pessoas) foi fator preditor para o início de uso de cannabis, na $8^{\mathrm{a}}$ série. Já, o consumo de tabaco, ser favorável ao álcool e a intenção futura do consumo de drogas ilícitas, na $8^{\text {a }}$ série, relacionaram-se ao início de consumo de cannabis na $9^{\text {a }}$ série. $\mathrm{O}$ início de consumo de cannabis na $10^{\mathrm{a}}$ série esteve associado a ser favorável ao tabaco, intenção futura do consumo de cannabis e gasto do tempo livre em bares e casas noturnas, na $9^{\text {a }}$ série. Em relação às meninas, os fatores preditores, quanto ao início do uso de cannabis, foram: cursar a $7^{\mathrm{a}}$ série, o uso do tabaco pelo grupo de pares, baixo rendimento escolar e gasto do tempo livre em bares ou em casas noturnas, no primeiro ano. Estudar em escola pública, morar com pais solteiros, consumir tabaco, consumo experimental de álcool e intenção futura de consumo de cannabis, na $8^{\text {a }}$ série, favoreceram o início de consumo na $9^{\mathrm{a}}$ série. O início de cannabis na $10^{\mathrm{a}}$ série esteve associado ao fato de estudarem em escola pública, consumo de tabaco, comportamento de risco no consumo de álcool, intenção futura de cannabis e comportamento antissocial.

Foi realizada, em 2007, uma revisão sistemática ${ }^{(28)}$ para verificar os fatores associados ao início do consumo de cannabis. Os resultados obtidos nessa revisão sugerem que os fatores associados para a iniciação de uso de cannabis são: ser do gênero masculino, consumir tabaco e álcool e possuir relação problemática com os pais. É importante salientar que a taxa de consumo de cannabis se iguala em ambos os sexos, principalmente se as meninas possuírem as mesmas oportunidades de consumo da substância, especialmente aquelas que iniciaram o uso de cannabis antes dos 21 anos de idade.

Este artigo de revisão apresenta um segundo estudo que verifica os fatores de iniciação do uso de cannabis especificamente nas meninas ${ }^{(29)}$. Os resultados revelam que o início do uso de cannabis esteve associado ao uso do álcool, tabaco e ser pertencente a grupo de pares que possuíam atitudes favoráveis ao uso de álcool, tabaco e cannabis, além de manifestar comportamentos impulsivos e desinibidos.

No que diz respeito à relação entre o consumo precoce de tabaco e álcool e os riscos de consumir cannabis, foi verificado, em pesquisa ${ }^{(30)} \mathrm{com} 54.001$ escolares com idade entre 8 e 20 anos, que o consumo de tabaco, na presença do consumo de álcool, foi fator preditor para o uso de cannabis. Em contrapartida, o início tardio de consumo de tabaco e álcool resultou em fator protetor para o uso de cannabis. A probabilidade de consumo de cannabis foi maior em fumantes diários do que em fumantes de final de semana. Dessa forma, o risco de consumo de cannabis se associou, significativamente, à idade de início de consumo de tabaco, à frequência de consumo de tabaco e ao consumo simultâneo de álcool. Os autores sugerem que as estratégias de prevenção deveriam se orientar para evitar o consumo precoce de tabaco em escolares.

\section{Discussão}

De acordo com a revisão aqui discutida, verifica-se que os principais fatores associados ao uso de cannabis na adolescência são: idade precoce de experimentação de álcool e tabaco, prejuízo na relação parental, baixo controle de self, amigos usuários, atitudes favoráveis ao consumo e comportamento agressivo.

É importante ressaltar que, embora seja evidente em muitos estudos a alta prevalência do uso de cannabis na adolescência, se encontrou número reduzido de estudos que investigaram os fatores associados ao início do uso de cannabis $^{(17-30)}$. O predomínio dos estudos desta revisão foi de publicações dos anos 2004, 2006 e 2007, de estudos de delineamento longitudinal, de base populacional. Foi realizado apenas um estudo de revisão sistemática e um estudo para testar modelo transteórico com o objetivo de medir a iniciação do uso de substâncias psicoativas. Cabe salientar que as amostras dos estudos foram expressivas, os estágios avaliados foram desde a puberdade, adolescência 
precoce, adolescência, adolescência tardia e jovens adultos, escolas de ensino primário e secundário, e as respectivas séries escolares.

Percebe-se o importante exame dos tipos de variáveis mensuradas em todos os estudos revisados, possibilitando, assim, a consistência da conclusão dos resultados. A avaliação do modelo transteórico para medir a iniciação do uso de substâncias contempla cada uma das variáveis utilizadas nos diversos estudos ${ }^{(19)}$.

Os cinco estudos onde se avaliaram os fatores de iniciação do uso de cannabis destacam-se pelos métodos de análises, sendo esses: pertencer ao gênero masculino, consumir tabaco e álcool, ter relação problemática com os pais e o consumo de cannabis por parte do grupo de $\operatorname{pares}^{(17-18,20,24,27)}$.

As variáveis individuais, testadas nos diversos estudos, reforçam a ideia de que jovens informais, despreocupados e com baixa integração de ego $^{(17)}$, com condutas antissociais ${ }^{(24,27-28)}$, possuindo atitudes favoráveis em relação ao uso de drogas lícitas e previsão futura de uso de drogas ilícitas ${ }^{(24)}$ estão fortemente associadas ao início de uso de cannabis. Vale salientar que adolescentes com bom controle do self tiveram adequada e competente trajetória acadêmica e grupo de pares com menos uso de substância e os adolescentes que usaram menos substâncias e o pior controle do self refere-se ao grupo de adolescentes que tiveram mais eventos de vida desviantes e pares com uso de substâncias ${ }^{(19)}$.

Em relação às variáveis familiares, cabe ressaltar que os estudos que investigaram as práticas parentais ${ }^{(17,19,23,28)}$, verificaram que o baixo envolvimento parental e o aumento da disciplina coersiva na escola primária estiveram associados ao risco de exposição de cannabis na adolescência e no jovem adulto.

O status marital materno foi investigado ${ }^{(25)}$, sendo que a mudança marital da mãe, ocorrida entre 5 e 14 anos, esteve associada significativamente ao aumento do risco para uso de cannabis, ressaltando que não é o status marital sozinho que influencia as crianças e adolescentes, mas a experiência da extensão do impacto da mudança marital materna. Em contrapartida, outros estudos reforçam que as crianças e adolescentes que tiveram morte parental antes dos $15^{(20)}$ e famílias de pais solteiros, divorciados e separados apresentaram associação significativa com o início de cannabis $^{(18,27)}$. O uso materno de substância ilícita e o nível de consumo de álcool estiveram diretamente associados ao uso tardio de cannabis. A idade precoce de início do uso de cannabis entre os adolescentes ${ }^{(25)}$ e o consumo parental de substâncias foram enfocados na maioria dos estudos ${ }^{(17,18,20,27,28)}$. É importante afirmar que os fatores preditores do início do uso de cannabis emergem durante a pré-adolescência, adolescência tardia e no adulto jovem $^{(17)}$, identificando que o uso precoce de cannabis na puberdade e na pré-adolescência é fator preditor para o uso regular de cannabis ${ }^{(18,20,22,26,30)}$, na adolescência tardia.

O uso de drogas lícitas aumenta significativamente a associação com as ilícitas. A escala de progressão das substâncias psicoativas é ordenada em um crescente de iniciação, onde, primeiramente vem o tabaco, álcool, cannabis e outras substâncias ilícitas. Assim, a experimentação ocorre normalmente através de substâncias psicoativas lícitas para as substâncias ilícitas ${ }^{(30)}$, e o uso precoce de substâncias é fator preditor para a iniciação da droga subsequente. O uso precoce de cannabis (antes dos 16 anos) esteve associado ao anterior uso de tabaco e álcool nas faixas de 12 a $14 \operatorname{anos}^{(26)}$.

Em todos os estudos revisados, o gênero masculino é preditor da incidência do uso e abuso de cannabis $^{(20,28)}$, enquanto isso, houve aumento na incidência de consumo entre as meninas, especialmente antes dos 21 anos de idade, bem como aumento da dependência entre as consumidoras $^{(28)}$. Ainda, os estudos referem que as meninas que tinham grupo de pares com atitudes favoráveis ao uso de álcool, tabaco e cannabis tiveram significativa associação com o início de uso de cannabis. Outro fator é a oportunidade e a disponibilidade das substâncias psicoativas. Sugerem os autores que, se as meninas tiveram mais acesso às substâncias, as taxas de uso, em ambos os sexos, tornam-se similares ${ }^{(28)}$.

Muitos autores sugerem que o grupo de pares tem forte influência na adolescência, visto que a pressão do grupo e atitudes favoráveis ao uso de substâncias psicoativas, bem como o uso propriamente dito ${ }^{(17,18,20,25,27)}$ de drogas entre os amigos e a opção pelo grupo no tempo livre $^{(27)}$, favorecendo a associação com o início de uso de cannabis.

Dos 14 estudos apenas um $^{(21)}$ testou a variável religiosidade em relação ao fator de início de uso de cannabis, Deve-se considerar que o número da amostra desse estudo é significativo $(\mathrm{n}=12.589)$, evidenciando que prática religiosa tem inversa associação com o uso precoce de tabaco e cannabis. Afirma ainda que jovens sem afiliação religiosa possuem duas ou três vezes mais chances de usar cannabis quando comparados aos jovens que possuem práticas católicas. Refere-se, ainda, que práticas religiosas representa fator de proteção ao uso precoce de tabaco e cannabis, mas esse comportamento não influencia na decisão de consumir a droga, uma vez que tenha tido oportunidade de uso.

Esses foram os estudos revisados. Torna-se necessário ressaltar as limitações metodológicas desta revisão, visto que a análise estatística não foi realizada com o objetivo de categorizar por metodologia cada estudo revisado. Fica como sugestão para os próximos trabalhos que se tenha como objetivo avaliar os fatores associados ao início do uso de cannabis.

Considerando a revisão das publicações nesses últimos anos, a respeito dos fatores associados ao início do uso de cannabis, pode-se observar que a investigação detalhada a respeito de cada fator, tanto de risco como de proteção, contribuirá para medidas preventivas de uso de drogas na adolescência. Para cada fator de risco para o início de cannabis, deve-se encontrar o equilíbrio do comportamento com um fator protetor, como, por exemplo, comportamento agressivo precoce, o fator protetor é o controle do self; deficiente supervisão parental, o fator protetor é adequado monitoramento parental; abuso de substância, o fator protetor é competência acadêmica; 
facilidade e disponibilidade de droga, o fator protetor é políticas antidrogas, nas comunidades empobrecidas, o fator de proteção pode ser equilibrado com fortes laços com a vizinhança.

\section{Conclusão}

Pelo exposto, os fatores associados ao início de uso de cannabis são: ser do gênero masculino, consumir tabaco e álcool em idade precoce, ter relação problemática com um dos pais, consumo de substâncias pelos pais, consumo de cannabis pelos pares e rede social desfavorável. Fica claro, em todas as investigações, que a iniciação, ocorrendo em idade precoce, aumenta a chance para desenvolver transtorno de uso de substâncias psicoativas. A progressão de uso e a escalada para outras substâncias irão interferir enormemente no desenvolvimento do adolescente, trazendo sérias consequências individuais, sociais, afetivas e ambientais. Torna-se necessário o cuidado com o consumo do álcool e o tabaco, no sentido de que o uso precoce dessas duas substâncias é preditor para o início do uso de cannabis. Cabe ressaltar que o álcool é droga ilícita antes dos 18 anos e a conscientização por parte da sociedade, no sentido de prevenção dessas duas substâncias, pode interferir na diminuição do uso de cannabis e de outras drogas subsequentes. Políticas públicas em relação ao uso de drogas lícitas diminuiriam o consumo dessas substâncias, assim como sua associação com outras substâncias, principalmente a cannabis. Em relação à família, o monitoramento parental precoce, na fase da puberdade, aumentaria a chance desse adolescente não fazer uso precoce de cannabis.

\section{Referências}

1. Pinsky I, Bessa M. Apresentação. In: Pinsky I, Bessa M. Adolescência e drogas. São Paulo: Contexto; 2004. p. 11-2. 2. Noto AR. Os índices de consumo de psicotrópicos entre os adolescentes no Brasil. In: Pinsky I, Bessa M. Adolescência e drogas. São Paulo: Editora Contexto; 2004. p. 45-53.

3. Andrade A, Duarte P, Oliveira L. I Levantamento nacional sobre uso de álcool, tabaco e outras drogas entre universitários das 27 capitais brasileiras; 2010. [acesso ago 2010]. Disponível em: htpp://www.obid.senad.gov.br 4. National Institute on Drug Abuse (NIDA). Marijuana. [acesso maio 2008]. Disponível em: http://www.drugabuse. gov/drugPages/Marijuana.html.

5. Monitoring the Future - National results on adolescent drug use - Overview of key Findings 2008. [acesso maio 2008]. Disponível em: http:// www.monitoringthefuture. org/pubs/monographs/overview2008.pdf.

6. Fergusson DM, Horwood LJ, Swain-Campell N. Cannabis use and psychosocial adjustment in adolescent and young adulthood. Addiction. 2002;97:1123-35.

7. Winters KC, Lee CY. Likelihood of developing an alcohol and cannabis use disorders during use disorders during youth: association with recent use and age. Drug Alcohol Depend. 2008; 92(1-3):239-47.
8. Galduróz JC, Noto AR, Fonseca AM \& Carlini EA. $\mathrm{V}$ Levantamento Nacional sobre o consumo de drogas psicotrópicas entre estudantes do ensino fundamental e médio da rede pública de ensino nas 27 capitais brasileiras 2004. São Paulo: UNIFESP; 2005.

9. Muza B, Muccillo, Barbieri. Consumo de substâncias psicoativas por adolescentes escolares de Ribeirão Preto, SP (Brasil). I - Prevalência do consumo por sexo, idade e tipo de substância. Rev Saúde Pública, 1997;31(1):21-9.

10. Baús J, Kupek E, Pires M. Prevalência e fatores de risco relacionados ao uso de drogas entre escolares. Rev Saúde Pública. 2002;36(1):40-6.

11. Saibro P, Ramos S. Consumo de substâncias psicoativas em estudantes de ensino médio e fundamental de escola pública e privada de Porto Alegre. In: $15^{\circ}$ Congresso da Associação Brasileira de Estudos sobre Álcool e Drogas; 2003. Setembro 3-7; São Paulo. Brasil; 2003.

12. Andrade TR, Argimoni IL. Sintomas depressivos e o uso de substâncias psicoativas durante a vida em adolescentes. Rev Bras Terapias Cognitivas. 2006;2(1):95-104.

13. Micheli D, Formigoni M. Drug use by Brazilian students: Associations with family, psychosocial, health, demografic and behavioral characteristics. Addiction. 2004;99(5):570-8.

14. Fergusson D, Horwwod L, Lynskey M, Madden P. Early reactions to cannabis predict later dependence. Arch Gen Psychiatry. 2003;60: 033-9.

15. Freitas C. Família: fator de risco ou proteção para o uso de drogas? In: Adolescência, drogas e o sistema de justiça. Projeto de Atenção especial ao adolescente infrator usuário de Drogas; 2003; Porto Alegre: PUCRS; 2003.

16. National Institute on Drug Abuse (NIDA). Preventing drug abuse among children and adolescents. Risk factors and protective factors. [acesso maio 2008]. Disponível em: http://www.drugabuse.gov/Prevention/risk.html.

17. Brook JS, Kessler RC, Cohen P. The onset of marijuana from preadolescence and early adolescence to young adulthood. Dev Psychopathol. 1999;11(4):901-14.

18. Coffey C, Lynskey M, Wolfe R, Patton. Initiation and progression of cannabis use in a population-base Australian adolescent longitudinal study. Addiction. 2000;95(11):1679-90.

19. Wills TA, Cleary S, Filer M, Shinar O, Mariani J, Spera K. Temperament related to early-onset substance use: test of a developmental model. Prev Sci. 2001;2(3):145-63.

20. Von Sindow K, Lieb R, Pfister H, Höfler M, Wittchen $\mathrm{Hu}$. What predicts incident use cannabis and progressive to abuse and dependence? A 4-year prospective examination of risk factors in community sample of adolescents and young adults. Drug Alcohol Depend. 2002;68(1):49-64.

21. Chen CY, Dormitzer, CM, Bejarano J, Anthony JC. Reliosity and the earliest stages of adolescent drug involviemnt in seven contries of Latin America. Am J Epidemiol. 2004; 159(12):1180-8.

22. Patton G, McMorris B, Toumbourou J, Hemphill S, Donath S, Catalano. Puberty and onset of substance use and abuse. Pediatrics. 2004;114(3):300-6. 
23. Chen CC, Storr C, Anthony J. Influences of parenting practices on the risk of having a chance to try cannabis. Pediatrics. 2005;115(6):1631-9.

24. Best D, Gross S, Manning V, Gossop M, Witton J, Strang J. Cannabis use in adolescents: the impact of risk and protective factors and social functioning. Drug Alcohol Rev. 2005;24(6): 483-8.

25. Hayatbakhsh M., Najman J, Jamrozik, Mamun A., Willians G, Alati R. Changes in maternal marital status are associated with young adults cannabis use: evidence from a 21-year follow-up of a birth cohort. Int J Epidemiol. 2006;35(3):673-9.

26. Agrawal A, Grant JD, Waldron M, Duncan AE, Scherer Jf, Lynskey MT, Madden PA, Bucholz KK, Heath AC. Risk for initiation of substance use as a function of age of onset of cigarette, alcohol and cannabis use: findings in a Midwestern female twin cohort. Prev Med.2006;43(2):125-8.

27. Guxens M, Nebot M, Ariza C, Ochoa D.. Factors associated with the onset of cannabis use: a systematic review of cohort studies. Gac Sanit. 2007;21(3):252-60.

28. Guxens M, Nebot M, Ariza C. Age and sex differences in factors associated with the onset of cannabis use: a cohort study. Drug Alcohol Depend. 2007;88 (2-3):234-43.

29. Agrawal, A, Grant JD, Waldron M, Duncan AE, Scherer JF, Lynskey MT, Maddeen, Bucholz KK, Heath AC. (2006). Correlates of cannabis in a longitudinal sample of young women the importance of peers influences. Prev Med. 2006;43(2):125-8.

30. Iglesias V, Cavada G, Silva C, Càceres D. Early tobacco and alcohol consumption as modifying risk factors on marijuana use. Rev Saúde Pública. 2007;41:4.

\section{Como citar este artigo:}

Andrade TMR, Ramos SP. Fatores de proteção e de risco associados ao início do uso de cannabis: revisão sistemática.

SMAD, Rev. Eletrônica Saúde Mental Álcool Drog. (Ed. port.). maio-ago. 2011 [acesso:

Disponível em: ];7(2):98-106.

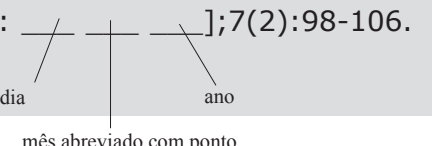

